\title{
From Physics to Living Systems- Applicable Mathematical Models
}

\section{Carlo Bianca*}

Polytechnic of Turin, Department of Mathematical Sciences, Corso Duca degli Abruzzi Torino (TO), Italy

The mathematical modeling of living systems has recently gained ground. Differently from physics systems, the living entities are capable of interacting among themselves and with their outer environment and are able to perform individual strategies that can modify the microscopic interactions among the entities composing the system. Living systems include, but are not limited, biological matter, crowds, traffic and swarms dynamics, opinion formation and economic systems.

Complexity is the common characteristic of these systems. The overall system exhibits behaviors and characteristics that are different from those of the elements constituting the system. Ultimately, the whole can be much more than the sum of its parts. Heterogeneity characterizes many living systems, e.g. in the biological systems case cells have different phenotypes generated by the same genotype. Living systems usually operate out-of-equilibrium, in fact for instance biological organisms are in a constant struggle with their environment to remain in a particular out-of-equilibrium state, namely alive. This dynamics is related to the search for well-being.

Moreover the study of living systems, in general of complex systems, always needs a multi scale approach. For instance, the dynamics of a cell at the molecular (genetic) level determines the cellular behaviors. Finally, the structures of the macroscopic tissues depend on such a dynamics. Ultimately, only one observation and representation scale is not sufficient to describe the dynamics of living systems. The interested reader in a deeper understanding of these systems is referred to the review paper [1] and book [2].

In the last three decades different mathematical methods and models have been developed for the modeling of living systems. Ordinary Differential Equations (ODE), Partial Differential Equations (PDE), kinetic theory approach, continuum mechanics approach, equilibrium and non equilibrium statistical mechanics are, among others, the most used frameworks. ODE-based models are employed for studying the time evolution of the density of the populations under consideration; PDE-based models deals with the evolution of systems with an internal structure (usually the age); kinetic theory models are concerned with the modeling of interactions among the entities; continuum mechanics approach is based on the fluid-assumption of the systems. Each framework is suitable for the modeling of the complex systems depending on what is needed to model.

Recently investigation on a new mathematical framework which couples kinetic theory models with non equilibrium statistical mechanics tools (Gaussian thermostat), the so-called thermos tatted kinetic theory, has been summarized in [3]. This new framework requires a much more spread ground of research that must be, at the same time, accurate and rigorous, and is proposed for the modeling of physics systems and living systems.

Although there has been much interest in developing and using mathematical models for complex living systems, the tuning with empirical data is a very hard problem and more investigations are needed in this direction. New effective methods or the proper combination of existing methods should be developed to reach this goal. In particular genetic algorithms has been applied with the aim to tune mathematical models with experimental data, see, among others, papers $[4,5]$. It is worth stressing that the OMICS' Applied \& Computational Mathematics Open Access policy (http://www. omicsonline.org/OpenAccess.php) and the special features provided by the publisher (http://www.omicsonline.org/special-features.php) can help the community to improve this research line.

\section{References}

1. Bianca C (2012) Thermostatted kinetic equations as models for complex systems in physics and life sciences. Physics of Life Reviews.

2. Bianca C, Bellomo N (2011) Towards a mathematical theory of complex biological systems. Series in mathematical biology and medicine. World Scientific.

3. Bianca C (2012) Onset of nonlinearity in thermostatted active particles models for complex systems. Nonlinear Analysis: Real World Applications 13: 2593-2608.

4. Bianca C, Pennisi M (2012) The triplex vaccine effects in mammary carcinoma: A nonlinear model in tune with simtriplex. Nonlinear Analysis: Real World Applications 13: $1913-1940$.

5. Pappalardo F, Lollini PL, Castiglione F, Motta S (2005) Modeling and simulation of cancer immunoprevention vaccine. Bioinformatics 21: 2891-2897.
*Corresponding author: Polytechnic of Turin, Department of Mathematical Sciences, Corso Duca degli Abruzzi Torino (TO), Italy, Tel: 39-011-090-7538, Fax: 39-011-090-7599, E-mail: carlo.bianca@polito.it

Received October 25, 2012; Accepted October 29, 2012; Published November 01, 2012

Citation: Bianca C (2012) From Physics to Living Systems- Applicable Mathematical Models. J Applied Computat Mathemat 1:e123. doi:10.4172/21689679.1000e123

Copyright: (c) 2012 Bianca C. This is an open-access article distributed under the terms of the Creative Commons Attribution License, which permits unrestricted use, distribution, and reproduction in any medium, provided the original author and source are credited. 